Zbigniew Grande, Institute of Mathematics, Pedagogical University, ul. Chodkiewicza 30, 85-064 Bydgoszcz, Poland. e-mail:

grande@wsp. bydgoszcz.pl

\title{
ON SOME SPECIAL NOTIONS OF APPROXIMATE QUASI-CONTINUITY
}

\begin{abstract}
Some special notions of approximate quasi-continuity and cliquishness are considered. Moreover, uniform, pointwise and transfinite convergence of sequences of such functions are investigated.
\end{abstract}

Let $\mathbb{R}$ be the set of all reals and let $\mu_{e}(\mu)$ denote outer Lebesgue measure (Lebesgue measure) in $\mathbb{R}$. Let

$$
\begin{aligned}
& d_{u}(A, x)=\limsup _{h \rightarrow 0^{+}} \mu_{e}(A \cap(x-h, x+h)) / 2 h \\
& \left(d_{l}(A, x)=\liminf _{h \rightarrow 0^{+}} \mu_{e}(A \cap(x-h, x+h)) / 2 h\right)
\end{aligned}
$$

denote the upper (lower) density of a set $A \subset \mathbb{R}$ at a point $x$. A point $x \in \mathbb{R}$ is called a density point of a set $A \subset \mathbb{R}$ if there exists a measurable (in the sense of Lebesgue) set $B \subset A$ such that $d_{l}(B, x)=1$. The family

$$
\mathcal{T}_{d}=\{A \subset \mathbb{R} ; A \text { is measurable and each } x \in A \text { is a density point of } A\}
$$

is a topology called the density topology [1].

Moreover, let $\mathcal{T}_{e}$ denote the Euclidean topology on $\mathbb{R}$. All considered functions will be real and defined on $\mathbb{R}$. A point $x$ is a continuity point (an approximate continuity point) of a function $f$ if it is a continuity point of $f$ considered as an application from $\left(\mathbb{R}, \mathcal{T}_{e}\right)\left(\left(\mathbb{R}, \mathcal{T}_{d}\right)\right)$ to $\left(\mathbb{R}, \mathcal{T}_{e}\right)$. Denote by $C(f)$ (resp. $A(f)$ ) the set of all continuity (approximate continuity) points of $f$. Define the following families of functions:

Key Words: continuity, strong quasicontinuity, density topology, oscillation, sequences of functions, uniform convergence.

Mathematical Reviews subject classification: 26A15, 54C08, 54C30

Received by the editors December 3, 1996 
- $f \in \mathcal{A}_{1}\left(f \in \mathcal{A}_{2}\right)$ if for every point $x$, for every positive real $\eta$, and for every set $A \in \mathcal{T}_{d}$ containing $x$ there is a point $u \in A \cap C(f)(u \in A(f) \cap A)$ such that $|f(u)-f(x)|<\eta$;

- $f \in \mathcal{A}_{3}\left(f \in \mathcal{A}_{4}\right)$ if for every point $x$, for every positive real $\eta$, and for every set $A \in \mathcal{T}_{d}$ containing $x$ there is an open interval $I$ such that $I \cap A \neq \emptyset, I \cap A \subset C(f)(I \cap A \subset A(f))$ and $|f(u)-f(x)|<\eta$ for all points $u \in I \cap A$;

- $f \in \mathcal{A}_{5}\left(f \in \mathcal{A}_{6}\right)$ if for every nonempty set $A \in \mathcal{T}_{d}$ there is an open interval $I$ such that $I \cap A \neq \emptyset$ and $I \cap A \subset C(f)(I \cap A \subset A(f))$.

- $f \in \mathcal{A}_{7}$ if for each positive real $\eta$ and for each nonempty set $A \in \mathcal{T}_{d}$ there is an open interval $I$ such that $I \cap A \neq \emptyset, I \cap A \subset A(f)$ and osc $f \leq \eta$ on $I \cap A$.

Moreover, a function $f$ is said to be strongly quasi-continuous [strongly cliquish] (abbreviated s.q.c. [s.c]) at a point $x$ if for every set $A \in \mathcal{T}_{d}$ containing $x$ and for every positive real $\eta$ there is an open interval $I$ such that $I \cap A \neq \emptyset$ and $|f(t)-f(x)|<\eta$ for all $t \in A \cap I$ [osc $f<\eta$ on $I \cap A]$ ([4]).

Let $f: \mathbb{R} \longrightarrow \mathbb{R}$ be a function and let $x \in \mathbb{R}$ be a point. If there is an open set $U$ such that $d_{u}(U, x)>0$ and the restricted function $f \mid(U \cup\{x\})$ is continuous at $x$, then $f$ is s.q.c. at $x$.

By elementary proofs, we obtain:

Remark 1. If all functions $f_{n}, n=1,2, \ldots$, of some uniformly converging sequence $\left(f_{n}\right)_{n}$ are s.q.c. at a point $x$, then its limit $f$ is also s.q.c. at $x$.

Remark 2. If $f \in \mathcal{A}_{i}, i=1,3,4$, then $f$ is s.q.c. at each point.

Remark 3. The inclusions

$$
\mathcal{A}_{3} \subset \mathcal{A}_{1} \subset \mathcal{A}_{2} ; \mathcal{A}_{3} \subset \mathcal{A}_{4} \subset \mathcal{A}_{2} ; \mathcal{A}_{3} \subset \mathcal{A}_{5} \cup \mathcal{A}_{4} \subset \mathcal{A}_{7} \subset \mathcal{A}_{6}
$$

are true.

Since every function which is s.q.c. at each point is also almost everywhere continuous [4], we can observe that a function $f \in \mathcal{A}_{1}$ if and only if $f$ is s.q.c. at each point $x$ and that the families $\mathcal{A}_{i}, i=1,3,4$, contain only almost everywhere continuous functions. It is obvious also that all functions belonging to the family $\mathcal{A}_{5}$ are almost everywhere continuous.

Approximately continuous functions are in Baire class 1, so they belong to $\mathcal{A}_{7} \cap \mathcal{A}_{2}$. Since there are approximately continuous functions which are not almost everywhere continuous ([1]), in the families $\mathcal{A}_{7}$ and $\mathcal{A}_{2}$ there are 
functions which are not almost everywhere continuous. However, if $f \in \mathcal{A}_{2} \cup$ $\mathcal{A}_{6}$, then for every positive real $\eta$ and for every measurable set $A$ with $\mu(A)>0$ there is a measurable subset $B \subset A$ such that $\mu(B)>0$ and osc $f \leq \eta$ on $B$. So, by Davies theorem $([2,3])$, every function $f \in \mathcal{A}_{2} \cup \mathcal{A}_{6}$ is measurable (in the sense of Lebesgue). If $f \in \mathcal{A}_{6}$, then for every open interval $I$ and for every positive real $\eta$ there is an open interval $J \subset I$ such that osc $f \leq \eta$ on $J$. Thus, the set $C(f)$ of arbitrary function $f \in \mathcal{A}_{6}$ is dense, and consequently it is a residual $G_{\delta}$ set. So, every function $f \in \mathcal{A}_{6}$ has the Baire property. However, there are functions $f \in \mathcal{A}_{2}$ which do not have the Baire property. Therefore, we adopt the following definition ([6]).

A function $f$ is called approximately quasi-continuous at a point $x$ if for every positive real $\eta$ and for each set $A \in \mathcal{T}_{d}$ containing $x$ there is a nonempty set $B \subset A$ belonging to $\mathcal{T}_{d}$ such that $|f(t)-f(x)|<\eta$ for all $t \in B$.

Observe that a function $f$ is approximately quasi-continuous at every point if and only if $f \in \mathcal{A}_{2}$. In [6] it is proved that for every measurable function $f$ there is a sequence of approximately quasi-continuous functions $f_{n}, n=$ $1,2, \ldots$ such that $f=\lim _{n \rightarrow \infty} f_{n}$. So, if $f$ is measurable without the Baire property, then there is an index $k$ such that the function $f_{k}$ does not have the Baire property. Such function $f_{k}$ is in the family $\mathcal{A}_{2}$ as an approximately quasi-continuous function, but it doesn't have the Baire property. From the above it follows that

$$
\mathcal{A}_{2} \backslash \mathcal{A}_{i} \neq \emptyset, \quad i=1,3,4,5,6,7 .
$$

Since there is an approximately continuous function $f$ with $\operatorname{int}(C(f))=\emptyset$ ([1]) we obtain $\mathcal{A}_{7} \backslash \mathcal{A}_{5} \neq \emptyset$.

The function $f(x)=0$ for $x \neq 0$ and $f(0)=1$ belongs to $\mathcal{A}_{5} \subset \mathcal{A}_{7}$, but it is not in $\mathcal{A}_{2} \supset \mathcal{A}_{1} \supset \mathcal{A}_{3}$. Moreover, it is not in $\mathcal{A}_{4}$. So,

$$
\mathcal{A}_{5} \backslash \mathcal{A}_{i} \neq \emptyset, \quad i=1,2,3,4 .
$$

If $g$ is an approximately continuous function such that $g(\mathbb{R})=[0,1]$ and the set $C(f)=g^{-1}(0)$ is dense and its interior is empty ([1]), then the function $f(x)=g(x)$ for $x \neq 0$ and $f(0)=2$ belongs to $\mathcal{A}_{7} \backslash \mathcal{A}_{i}$ for $i=1,2,3,4,5$.

As an example of function $f \in \mathcal{A}_{1} \backslash \mathcal{A}_{6}$ we can take any strictly monotone function which is continuous from the right at every point and which is such that the set $\mathbb{R} \backslash C(f)$ is dense.

So, we obtain the following assertion.

Theorem 1. The inclusions

$$
-\mathcal{A}_{3} \subset \mathcal{A}_{1} \subset \mathcal{A}_{2} ; \quad \mathcal{A}_{3} \subset \mathcal{A}_{4} \subset \mathcal{A}_{2} ;
$$


$-\mathcal{A}_{3} \subset \mathcal{A}_{5} \subset \mathcal{A}_{7} ; \quad \mathcal{A}_{3} \subset \mathcal{A}_{4} \subset \mathcal{A}_{7} ;$

are true. Moreover, each of the above inclusions is strict and

$$
\mathcal{A}_{1} \backslash \mathcal{A}_{6} \neq \emptyset \text {. }
$$

The inclusion $\mathcal{A}_{7} \subset \mathcal{A}_{6}$ is evident.

Theorem 2. The relation $\mathcal{A}_{6} \backslash \mathcal{A}_{7} \neq \emptyset$ is true.

Proof. Let $A_{1} \subset I_{1}=(0,1)$ be a Cantor set of positive measure. In the second step in every component $I_{1, n}, n=1,2, \ldots$, of the set $I_{1} \backslash A_{1}$ we find an open interval $J_{1, n}$ having the same centers as $I_{1, n}$ and such that $\left|J_{1, n}\right|<$ $4^{-2}\left|I_{1, n}\right|$, where the symbol $\left|J_{1, n}\right|$ denotes the length of the interval $J_{1, n}$. Next, in each open interval $J_{1, n}$ we find a Cantor set $A_{1, n} \subset J_{1, n}$ of positive measure.

In general, in the $k^{t h}$ step $(k>2)$ we consider all components $I_{1, n_{2}, \ldots, n_{k}}$ of the set $J_{1, n_{2}, \ldots, n_{k-1}} \backslash A_{1, n_{2}, \ldots, n_{k-1}}, n_{i} \geq 1$ for $1<i \leq k$, and we find open intervals $J_{1, n_{2}, \ldots, n_{k}} \subset I_{1, n_{2}, \ldots, n_{k}}$ having the same centers as $I_{1, n_{2}, \ldots, n_{k}}$ and such that

$$
\left|J_{1, n_{2}, \ldots, n_{k}}\right|<4^{-k}\left|I_{1, n_{2}, \ldots, n_{k}}\right| .
$$

For $1<i \leq k$ and $n_{i}=1,2, \ldots$, let $A_{1, n_{2}, \ldots, n_{k}} \subset J_{1, n_{2}, \ldots, n_{k}}$ be a Cantor set of positive measure and let

$$
B_{1, n_{2}, \ldots, n_{k}}=\left\{x \in A_{1, n_{2}, \ldots, n_{k}} ; d_{l}\left(A_{1, n_{2}, \ldots, n_{k}}, x\right)=1\right\} .
$$

Let $f(x)=1$ for $x \in B_{1, n_{2}, \ldots, n_{k}}$, whenever $k$ is even and $n_{i} \geq 1$ for $1<i \leq k$ and let $f(x)=0$ otherwise on $\mathbb{R}$. We shall prove that $f \in \mathcal{A}_{6}$. Let $A \in \mathcal{T}_{d}$ be a nonempty set. Denote by $B$ the union of all sets $B_{1, n_{2}, \ldots, n_{k}}$, where $k=2,3, \ldots$ and $n_{i}=1,2, \ldots$ for $1<i \leq k$. Observe that $B \subset A(f)$. So, if $A \subset B$, then for every open interval $I$ such that $I \cap A \neq \emptyset$ we have $I \cap A \subset A(f)$. If $A$ is not a subset $B$, then there is a point $x \in A \backslash B$. If $x$ is not in $\operatorname{cl}(B)$, where $\operatorname{cl}(B)$ denotes the closure of the set $B$, then $f$ is continuous at $x$ and for every interval $I \subset \mathbb{R} \backslash \operatorname{cl}(B)$ containing $x$ we obtain that $I \cap A \neq \emptyset$ and $I \cap A \subset C(f) \subset A(f)$. So, we suppose that $x \in \operatorname{cl}(B)$. If $x=0$ or $x=1$, then $f$ is unilaterally continuous at $x$ and there is an open interval $I \subset \mathcal{R} \backslash[0,1]$ with $I \cap A \neq \emptyset$. Consequently, in this case we have $f(t)=f(x)=0$ for all $t \in I \cap A$. If there are indexes $n_{2}, \ldots, n_{k}$ such that $x \in A_{1, n_{2}, \ldots, n_{k}} \backslash B_{1, n_{2}, \ldots, n_{k}}$, then, by (1), there is an open interval

$$
I \subset J_{1, n_{2}, \ldots, n_{k}} \backslash A_{1, n_{2}, \ldots, n_{k}} \backslash \bigcup_{n_{k+1}=1}^{\infty} J_{1, n_{2}, \ldots, n_{k+1}}
$$


such that $I \cap A \neq \emptyset$ and $I \cap A \subset C(f) \subset A(f)$. If not, there is a sequence of indexes $n_{2}, \ldots, n_{k}, \ldots$, such that

$$
x \in \bigcap_{k=1}^{\infty} I_{1, n_{2}, \ldots, n_{k}}=\bigcap_{k=1}^{\infty} J_{1, n_{2}, \ldots, n_{k}} .
$$

Since $d_{l}(A, x)=1$, there is an index $m$ such that for each $k \geq m$ we have

$$
\mu\left(A \cap I_{1, n_{2}, \ldots, n_{k}}\right)>\left|I_{1, n_{2}, \ldots, n_{k}}\right| / 2 .
$$

By (1), there is an index $j>m$ such that for each $k \geq j$ we obtain

$$
4\left|J_{1, n_{2}, \ldots, n_{k}}\right|<\left|I_{1, n_{2}, \ldots, n_{k}}\right| .
$$

Fix $k>j$ and observe that from (2) and (3) it follows that

$$
A \cap\left(I_{1, n_{2}, \ldots, n_{k}} \backslash \operatorname{cl}\left(J_{1, n_{2}, \ldots, n_{k}}\right)\right) \neq \emptyset .
$$

Consequently, there is an open interval $I$ such that $A \cap I \neq \emptyset$ and $I \cap A \subset$ $C(f) \subset A(f)$. So, $f \in \mathcal{A}_{6}$.

For the proof that $f$ is not in $\mathcal{A}_{7}$ it suffices to observe that $B \in \mathcal{T}_{d}$ and for every open interval $I$ with $I \cap B \neq \emptyset$ the oscillation osc $f$ on $I \cap B$ is greater than $a$ for every positive real $a<1$.

Remark 4. Observe that if $f \in \mathcal{A}_{6}$ is of the first Baire class, then $f \in \mathcal{A}_{7}$. Consequently, every approximately continuous function belongs to $\mathcal{A}_{7}$.

For a family $\Phi$ let $\mathcal{B}(\Phi)\left(\mathcal{B}_{u}(\Phi)\right)$ denote the family of all limits of converging (of uniformly converging) sequences of functions from $\Phi$. Moreover, let $Q_{s}$ denote the family of all functions which are s.q.c. at every point. By Remark 1 the family $Q_{s}=\mathcal{A}_{1}$ is uniformly closed.

Theorem 3. The equality $\mathcal{B}_{u}\left(\mathcal{A}_{3}\right)=Q_{s}$ is true.

Proof. Let $f \in Q_{s}$ be a function. It suffices to prove that for every positive real $\eta$ there is a function $g \in \mathcal{A}_{3}$ such that $|f-g| \leq \eta$. Fix a real $\eta>0$. Let

$$
E=\left\{y ; \mu\left(c l\left(f^{-1}(y)\right)\right)>0\right\} .
$$

Since $f$ is almost everywhere continuous, the set $E$ is countable. There is a sequence $\left(c_{k}\right)_{k=-\infty}^{\infty}$ of reals $c_{k} \in \mathbb{R} \backslash E$ such that $0<c_{k+1}-c_{k}<\eta / 2$ for all integers $k$ and

$$
\mathbb{R}=\bigcup_{k=-\infty}^{\infty}\left[c_{k}, c_{k+1}\right) .
$$


If $x$ is such that $c_{k} \leq f(x)<c_{k+1}$, then let $h(x)=c_{k}$. If $h$ is not s.q.c. at $x$ and $h(x)=c_{k}$, then we put $g(x)=c_{k-1}$. Otherwise let $g(x)=h(x)$. Since $f$ is s.q.c. at each point $x \in \mathbb{R}$, the function $g$ is the same on $\mathbb{R}$. But the image $g(\mathbb{R})$ is a discrete set, so we obtain $g \in \mathcal{A}_{3}$. Evidently, $|f-g| \leq \eta$.

Since $\mathcal{A}_{3} \subset \mathcal{A}_{4} \subset Q_{s}$, we obtain the following.

Corollary 1. The equality $\mathcal{B}_{u}\left(\mathcal{A}_{4}\right)=Q_{s}$ is true.

Remark 5. The equality $\mathcal{B}_{u}\left(\mathcal{A}_{2}\right)=\mathcal{A}_{2}$ is true.

Proof. Fix $f \in \mathcal{B}_{u}\left(\mathcal{A}_{2}\right)$. There is a sequence of functions $f_{n} \in \mathcal{A}_{2}, n=$ $1,2, \ldots$, which converges uniformly to $f$. Fix a point $x$, a positive real $\eta$ and a set $A \in \mathcal{T}_{d}$ containing $x$. Let $k$ be an index for which $\left|f_{k}-f\right|<\eta / 4$. Since $f_{k} \in \mathcal{A}_{2}$, there is a point $u \in A \cap A\left(f_{k}\right)$ such that $\left|f_{k}(u)-f_{k}(x)\right|<\eta / 4$. But $u \in A\left(f_{k}\right)$, so there is a measurable subset $B \subset A$ such that $\mu(B)>0$ and $\left|f_{k}(t)-f_{k}(u)\right|<\eta / 4$ for each point $t \in B$. The function $f$ is measurable as the limit of the sequence of measurable functions $f_{n}$. Consequently, there is a point $w \in B \cap A(f) \subset A \cap A(f)$. Moreover, we have

$$
\begin{aligned}
|f(w)-f(x)| \leq & \left|f(w)-f_{k}(w)\right|+\left|f_{k}(w)-f_{k}(u)\right|+\left|f_{k}(u)-f_{k}(x)\right| \\
& +\left|f_{k}(x)-f(x)\right|<\eta / 4+\eta / 4+\eta / 4+\eta / 4=\eta .
\end{aligned}
$$

Let $\mathcal{C}_{a e}$ denote the family of all functions $f$ for which $\mu(\mathbb{R} \backslash C(f))=0$. We have the following.

Remark 6. The equality $\mathcal{B}_{u}\left(\mathcal{A}_{5}\right)=\mathcal{C}_{a e}$ is true.

Proof. It suffices to prove that for every $f \in \mathcal{C}_{a e}$ and for every positive real $\eta$ there is a function $h \in \mathcal{A}_{5}$ with $|f-h| \leq \eta$. Fix $f \in \mathcal{C}_{a e}$ and a positive real $\eta$. Define the function $h$ the same as that in the proof of Theorem 4 and observe that $h \in \mathcal{A}_{5}$, because it is almost everywhere continuous and its image $h(\mathbb{R})$ is a discrete set.

Theorem 4. A function $f \in \mathcal{B}_{u}\left(\mathcal{A}_{7}\right)$ if and only if it is strongly cliquish at each point $x \in \mathbb{R}$.

Proof. Assume, to the contrary, that there is a function $f \in \mathcal{B}_{u}\left(\mathcal{A}_{7}\right)$ such that there are a nonempty set $A \in \mathcal{T}_{d}$ and a positive real $\eta$ with osc $f>\eta$ on $A \cap I$ for every open interval $I$ such that $I \cap A \neq \emptyset$. Since $f \in \mathcal{B}_{u}\left(\mathcal{A}_{7}\right)$, there is a function $g \in \mathcal{A}_{7}$ such that $|f-g|<\eta / 4$. For every open interval $I$ such that $I \cap A \neq \emptyset$ we obtain that osc $g>\eta / 2$ on the set $I \cap A$. But $g \in \mathcal{A}_{7}$, so there is an open interval $I$ such that $I \cap A \neq \emptyset$ and $I \cap A \subset A(g)$ and osc $g<\eta / 2$ 
on $I \cap A$. This contradiction shows that if $f \in \mathcal{B}_{u}\left(\mathcal{A}_{7}\right)$, then $f$ is s.c. at each point.

Now, suppose the function $f$ is s.c. at each point $x \in \mathbb{R}$. Fix a positive real $\eta$. We shall prove that there is a function $g \in \mathcal{A}_{7}$ such that $|f-g|<\eta$. Let $I_{1}$ be an open interval with rational endpoints such that osc $f<\eta$ on $I_{1}$. Fix an ordinal number $\alpha>1$ and suppose that for every ordinal number $\beta<\alpha$ there is an open interval $I_{\beta}$ with rational endpoints such that $\mu\left(I_{\beta} \backslash G_{\beta}\right)>0$, where $G_{\beta}=\cup_{\gamma<\beta} I_{\gamma}$, and osc $f<\eta$ on the set

$$
H_{\beta}=\left\{x \in I_{\beta} \backslash G_{\beta} ; d_{l}\left(I_{\beta} \backslash G_{\beta}, x\right)=1\right\} .
$$

Since the function $f$ is s.c. at every point, there is an open interval $I_{\alpha}$ with rational endpoints such that $\mu\left(I_{\alpha} \backslash G_{\alpha}\right)>0$ and osc $f<\eta$ on the set $H_{\alpha}$. By transfinite induction we find a transfinite sequence of such open intervals $\left(I_{\alpha}\right)_{\alpha<\alpha_{0}}$ with rational endpoints, where $\alpha_{0}$ is the first ordinal number for which $\mu\left(\mathbb{R} \backslash G_{\alpha_{0}}\right)=0$. Since the family of all open intervals with rational endpoints is countable, the ordinal number $\alpha_{0}$ is also countable. For every $\alpha<\alpha_{0}$ we find a point $x_{\alpha} \in H_{\alpha}$ and let $g(x)=f\left(x_{\alpha}\right)$ for $x \in H_{\alpha}$ and $g(x)=f(x)$ otherwise on $\mathbb{R}$. If $x \in H_{\alpha}$, then

$$
|f(x)-g(x)|=\left|f(x)-f\left(x_{\alpha}\right)\right|<\eta .
$$

In the remaining case $f(x)=g(x)$, so $|f-g|<\eta$. For the completeness of the proof it suffices to show that the function $g \in \mathcal{A}_{7}$. For this, fix a nonempty set $A \in \mathcal{T}_{d}$ and a positive real $\varepsilon$. There is an ordinal number $\beta<\alpha_{0}$ such that $I_{\beta} \cap A \neq \emptyset$ and $I_{\alpha} \cap A=\emptyset$ for $\alpha<\beta$. Then $\emptyset \neq A \cap I_{\beta} \subset H_{\beta}$, since otherwise we have $G_{\beta} \cap A \neq \emptyset$, a contradiction. Consequently, $g(x)=f\left(x_{\beta}\right)$ for each point $x \in I_{\beta} \cap A$. So, osc $f=0<\varepsilon$ on $I_{\beta} \cap A$.

Remark 7. The function $f$ from the proof of Theorem 2 is such that $f \in$ $\mathcal{A}_{6} \backslash \mathcal{B}_{u}\left(\mathcal{A}_{7}\right)$, since osc $f=1$ on the sets $I \cap B$ for every open interval I with $I \cap B \neq \emptyset$.

Observe that if $f \in \mathcal{B}_{u}\left(\mathcal{A}_{6}\right)$, then $f$ is measurable and the set $\mathbb{R} \backslash C(f)$ is of the first category. Moreover, if

$$
\operatorname{ap}-\operatorname{osc} f(x)=\inf \left\{\operatorname{osc}_{A} f ; \emptyset \neq A \in \mathcal{T}_{d}, \quad x \in A\right\},
$$

then we have the following.

Theorem 5. If a function $f$ belongs to $\mathcal{B}_{u}\left(\mathcal{A}_{6}\right)$, then for every positive real $\eta$ and for every nonempty set $A \in \mathcal{T}_{d}$ the set $\{x \in A$; ap-osc $f(x) \geq \eta\}$ is nowhere dense in $A$. 
Proof. Assume, to the contrary, that there are a function $f \in \mathcal{B}_{u}\left(\mathcal{A}_{6}\right)$, a nonempty set $A \in \mathcal{T}_{d}$, and a positive real $\eta$ such that for every open interval $J$ with $J \cap A \neq \emptyset$ there is a point $x \in J \cap A$ at which ap-osc $f(x) \geq \eta$. Since $f \in B_{u}\left(\mathcal{A}_{6}\right)$, there is a function $g \in \mathcal{A}_{6}$ such that $|f-g|<\eta / 3$. Observe that if ap-osc $f(x) \geq \eta$, then ap-osc $g(x) \geq \eta / 4$. So, for every open interval $J$ with $J \cap A \neq \emptyset$ there is a point $x \in J \cap A$ at which ap-osc $g(x) \geq \eta / 4$. But $g \in \mathcal{A}_{6}$, so there is an open interval $J$ with $J \cap A \neq \emptyset$ and $J \cap A \subset A(f)$. Consequently, for each point $x \in J \cap A$ we obtain ap-osc $g(x)=0<\eta / 4$, a contradiction.

Problem. Characterize the class $\mathcal{B}_{u}\left(\mathcal{A}_{6}\right)$.

Remark 8. Since every function $f \in \mathcal{A}_{2}$ is measurable and every measurable function is the limit of a sequence of approximately quasi-continuous functions (which belong to $\mathcal{A}_{2}$ ) ([6]), we obtain that $\mathcal{B}\left(\mathcal{A}_{2}\right)$ is the family of all measurable functions.

In [8] Mauldin shows that $f \in \mathcal{B}\left(\mathcal{C}_{a e}\right)$ if and only if there are a function $g$ of Baire class 1 and an $F_{\sigma}$-set $A$ of measure zero such that $\{x ; f(x) \neq g(x)\} \subset A$.

Theorem 6. The equality $\mathcal{B}\left(\mathcal{A}_{5}\right)=\mathcal{B}\left(\mathcal{C}_{a e}\right)$ is true.

Proof. Since every $f \in \mathcal{A}_{5}$ belongs to $\mathcal{C}_{a e}$, by Mauldin's theorem we obtain the inclusion $\mathcal{B}\left(\mathcal{A}_{5}\right) \subset \mathcal{B}\left(\mathcal{C}_{a e}\right)$. Let $f \in \mathcal{B}\left(\mathcal{C}_{a e}\right)$ be a function. By Mauldin's theorem there are a function $g$ of the first class of Baire and an $F_{\sigma}$-set $A$ of measure zero such that $\{x ; f(x) \neq g(x)\} \subset A$. Let $h=f-g$. Then $h(x)=0$ for each point $x$ which is not in $A$. There are closed sets $A_{n}, n=1,2, \ldots$, such that $A_{1} \subset \ldots \subset A_{n} \subset \ldots$ and $A=\bigcup_{n} A_{n}$. For $n=1,2, \ldots$ let $h_{n}(x)=h(x)$ for $x \in A_{n}$ and let $h(x)=0$ otherwise on $\mathbb{R}$. Since every set $A_{n}, n=1,2, \ldots$, is closed and of measure zero, it is nowhere dense and consequently every function $h_{n} \in \mathcal{A}_{5}, n=1,2, \ldots$. For the function $g$ there is a sequence of continuous functions $g_{n}, n=1,2, \ldots$, such that $g=\lim _{n \rightarrow \infty} g_{n}$. Observe that every function $f_{n}=g_{n}+h_{n}, n=1,2, \ldots$, belongs to $\mathcal{A}_{5}$ as the sum of the continuous function $g_{n}$ and the function $h_{n}$ belonging to $\mathcal{A}_{5}$. Since

$$
f=g+h=\lim _{n} g_{n}+\lim _{n} h_{n}=\lim _{n} f_{n},
$$

the proof is complete.

In [5] the following theorem is proved.

Theorem 7. Let $f$ be a function such that there is a Baire 1 function $g$ such that for every positive real $\eta$ and for each point $x$ such that $|f(x)-g(x)| \geq \eta$ there is a closed interval $I(x)$ containing $x$ and such that $\mu(I(x) \backslash \operatorname{cl}(\{t ; \mid f(t)-$ $g(t) \mid \geq \eta\}))=0$. Then there is a sequence of functions $f_{n} \in Q_{s}$ such that $\lim _{n} f_{n}=f$. 
Since every function $g$ from $Q_{s}$ is the limit of a sequence of functions from $\mathcal{A}_{3}$ which uniformly converges to $g$, every function satisfying the hypothesis of the above theorem belongs to $\mathcal{B}\left(\mathcal{A}_{3}\right)$.

We can prove the following.

Theorem 8. Let $f$ be a function such that there are a Baire 1 function $g$ and an $F_{\sigma}$ set $B$ of measure zero such that $\{t ; f(t) \neq g(t)\} \subset B$ and for every positive real $\eta$ and for each point $x$ such that $\mid f x)-g(x) \mid \geq \eta$ the upper density $d_{u}(\operatorname{cl}(\{t ;|f(t)-g(t)| \geq \eta\}), x)=0$. Then there is a sequence of functions $f_{n} \in \mathcal{A}_{3}, n=1,2, \ldots$, which converges to $f$.

Proof. Let $h=f-g$ and let $B=\cup_{n} B_{n}$, where every set $B_{i}$ is closed and $B_{i} \subset B_{i+1}$ for $i=1,2, \ldots$ For $n=1,2, \ldots$ let $A_{n}=\{x ;|h(x)| \geq 1 / n\}$. Fix a positive integer $n$. By our hypothesis there is a family of disjoint closed intervals $I_{k, l, i}, k \leq n, l, i=1,2, \ldots$ such that

- $I_{1, l, i} \subset \mathbb{R} \backslash\left(\operatorname{cl}\left(A_{n}\right) \cap B\right)$ for $l, i \geq 1$;

- $I_{k, l, i} \subset \mathbb{R} \backslash \operatorname{cl}\left(A_{n}\right)$ for $1<k \leq n, l, i=1,2, \ldots$;

- for each $k \leq n$ the inclusion

$$
I_{k, l, i} \subset A\left(\operatorname{cl}\left(A_{k}\right) \cap B_{k}, 1 / k\right)\left(=\left\{t ; \inf \left\{|u-t| ; u \in \operatorname{cl}\left(A_{k}\right) \cap B_{k}\right\} \leq 1 / n\right\}\right.
$$

is true for $l, i \geq 1$;

- if $k \leq n, l \geq 1$ and $x \in \operatorname{cl}\left(A_{k}\right) \cap B_{k}$, then $d_{u}\left(\bigcup_{i=1}^{\infty} I_{k, l, i}, x\right)>0$;

- for each $k \leq n$ and for each $x \in \mathbb{R} \backslash\left(A_{k} \cap B_{k}\right)$ there is an open set $U$ containing $x$ such that the set $\left\{(k, l, i) ; U \cap I_{k, l, i} \neq \emptyset\right\}$ is finite.

Next, in every interval $\operatorname{int}\left(I_{k, l, i}\right), k \leq n, l, i=1,2, \ldots$, we find a closed interval $J_{k, l, i}$ such that if $k \leq n, l \geq 1$ and $x \in \operatorname{cl}\left(A_{k}\right) \cap B_{k}$, then $\left.d_{u}\left(\bigcup_{i=1}^{\infty} J_{k, l, i}, x\right)\right)>0$. Let $\left(w_{1, l}\right)_{l=1}^{\infty}$ be a sequence of all rationals with $w_{1,1}=0$ and for $k>1$ let $\left(w_{k, l}\right)_{l=1}^{\infty}$ be a sequence of all rationals belonging to the interval $[-1 /(k-$ 1), $1 /(k-1)]$ with $w_{k, 1}=0$. Put

$$
h_{n}(x)= \begin{cases}w_{k, l} & x \in J_{k, l, i}, k \leq n, l, i \geq 1 \\ h(x) & x \in A_{n} \cap B_{n} \\ \text { linear on the components } & \\ \text { of the sets } I_{k, l, i} \backslash J_{k, l, i}, & l, i \geq 1, k \leq n \\ 0 & \text { otherwise on } \mathbb{R} .\end{cases}
$$


Evidently, the function $h_{n}$ is continuous at each point $x$ which is not in $\operatorname{cl}\left(A_{n}\right) \cap$ $B_{n}$. Fix a positive real $\eta$, a point $x \in \operatorname{cl}\left(A_{n}\right) \cap B_{n}$ and a set $A \in \mathcal{T}_{d}$ containing $x$. If there is an integer $k \leq n$ such that $x \in A_{k} \cap B_{k}$, then there is a rational $w_{k, l}$ such that $\left|h(x)-w_{k, l}\right|<\eta$. Since $d_{u}\left(\bigcup_{i} J_{k, l, i}, x\right)>0$, there is an interval $J_{k, l, i}$ such that $J_{k, l, i} \cap A \neq \emptyset$. Every point $t \in A \cap J_{k, l, i}$ is a continuity point of $h_{n}$ and

$$
\left|h_{n}(t)-h_{n}(x)\right|=\left|w_{k, l}-h(x)\right|<\eta .
$$

In the remaining case we obtain that $h_{n}(x)=0$ and $x \in \operatorname{cl}\left(A_{k}\right) \cap B_{k}$ for some positive integer $k$. Since $w_{k, 1}=0$ and since $d_{u}\left(\bigcup_{i} J_{k, 1, i}, x\right)>0$, there is an interval $J_{k, 1, i}$ such that $J_{k, 1, i} \cap A \neq \emptyset$. For each point $t \in J_{k, 1, i} \cap A$ the function $h_{n}$ is continuous at $t$ and $\left|h_{n}(t)-h_{n}(x)\right|=0<\eta$. So, $h_{n} \in \mathcal{A}_{3}$.

Now we will prove that $\lim _{n \rightarrow \infty} h_{n}=h$. If there is a positive integer $n$ with $x \in A_{n} \cap B_{n}$, then $h_{k}(x)=h(x)$ for $k \geq n$. If not, we have $h(x)=0$. Fix a positive real $\eta$ and a positive integer $n$ with $1 / n<\eta$. If $x \in \operatorname{cl}\left(A_{m}\right) \cap B_{m}$ for some positive integer $m$, then $h_{k}(x)=0$ for all $k \geq m$. So, we suppose that $x$ is not in $\operatorname{cl}\left(A_{m}\right) \cap B_{m}$ for $m \geq 1$. Since $x$ is not in the set $\operatorname{cl}\left(A_{n}\right) \cap B_{n}$, there is a positive integer $m>n$ such that $|x-y|>1 / m$ for every point $y \in \operatorname{cl}\left(A_{n}\right)$. Consequently,

$$
h_{k}(x) \leq 1 /(m-1) \leq 1 / n<\eta
$$

for every $k \geq m$. This completes the proof that $h=\lim _{n \rightarrow \infty} h_{n}$.

Since $g$ is a Baire 1 function, there is a sequence of continuous functions $g_{n}$ such that $g=\lim _{n \rightarrow \infty} g_{n}$. Evidently, the functions $f_{n}=h_{n}+g_{n}, n=1,2, \ldots$, belong to the family $\mathcal{A}_{3}$ and

$$
\lim _{n \rightarrow \infty} f_{n}=\lim _{n \rightarrow \infty} h_{n}+\lim _{n \rightarrow \infty} g_{n}=h+g=f .
$$

Corollary 2. If the function $f: \mathbb{R} \longrightarrow \mathbb{R}$ is almost everywhere continuous, then $f \in \mathcal{B}\left(\mathcal{A}_{3}\right)$.

Denote by $\mathcal{P}_{s}$ the family of all functions which are s.c. at each point.

Problem. Is it true that $\mathcal{P}_{s} \cap \mathcal{B}\left(\mathcal{C}_{a e}\right)=\mathcal{B}\left(\mathcal{A}_{3}\right)$ ?

Now, we will investigate the transfinite convergence of sequences. Let $\omega_{1}$ denote the first uncountable ordinal number. A transfinite sequence of functions $f_{\alpha}, \alpha<\omega_{1}$, converges to a function $f\left(\lim _{\alpha} f_{\alpha}=f\right)$ if for each point $x$ there is an ordinal number $\beta<\omega_{1}$ such that $f_{\alpha}(x)=f(x)$ for each countable ordinal $\alpha>\beta$.

Theorem 9. Let $\mathcal{K}$ be a family of functions such that if a function $f$ is not in $\mathcal{K}$, then there is a countable set $A$ such that for every function $g \in \mathcal{K}$ there is a point $x \in A$ with $g(x) \neq f(x)$. Then the limits of all converging transfinite sequences of functions from the family $\mathcal{K}$ belong to $\mathcal{K}$. 
Proof. Let $\left(f_{\alpha}\right) \in \mathcal{K}, \alpha<\omega_{1}$, and let $\lim _{\alpha} f_{\alpha}=f$. Suppose, to the contrary, that $f$ is not in $\mathcal{K}$. Then there is a countable set $A=\left\{x_{1}, x_{2}, \ldots\right\}$ such that for each function $g \in \mathcal{K}$ there is a point $x \in A$ with $g(x) \neq f(x)$. For each positive integer $n$ there is a countable ordinal number $\beta_{n}$ such that $f_{\alpha}\left(x_{n}\right)=f\left(x_{n}\right)$ for $\beta_{n}<\alpha<\omega_{1}$. There is a countable ordinal number $\beta$ such that $\beta_{n}<\beta$ for all positive integers $n$. So, $f_{\beta}\left(x_{n}\right)=f\left(x_{n}\right)$ for $n=1,2, \ldots$. Since $f_{\beta} \in \mathcal{K}$, we obtain a contradiction.

Remark 9. Observe that the families $P_{s}$ and $\mathcal{A}_{i}, i=1,3,5$, satisfy the hypothesis of the above Theorem 9.

Proof. If $f$ is not in $\mathcal{A}_{i}, i=1$ or 3 or 5 , then every countable set $A$ such that the set $\{(x, f(x)) ; x \in A\}$ is dense in the graph of the function $f$ satisfies all requirements.

Theorem 10. Assume the Continuum Hypothesis HC. For every function $f$ there is a transfinite sequence of functions $f_{\alpha} \in \mathcal{A}_{2}, \alpha<\omega_{1}$, such that $f=\lim _{\alpha} f_{\alpha}$.

Proof. Let $\left(x_{\alpha}\right)_{\alpha<\omega_{1}}$ be a transfinite sequence of all reals numbers. Fix an ordinal number $\alpha<\omega_{1}$ and let $\left(t_{n}\right)_{n}$ be a sequence of all numbers $x_{\beta}$ with $\beta \leq \alpha$ such that $t_{i} \neq t_{j}$ for $i \neq j, i, j=1,2, \ldots$. For every positive integer $n$ there are closed intervals $I_{n}, J_{n}$ and a closed set $A_{n}$ such that

$$
\begin{aligned}
& -t_{n} \text { is an endpoint of } I_{n} \text { and } J_{n} ; \\
& -J_{n} \subset I_{n} \text { and }\left|J_{n}\right|=\left|I_{n}\right| / 2 ; \\
& -A_{n} \subset J_{n} \backslash\left\{t_{k} ; k \neq n \text { and } k=1,2, \ldots\right\} ; \\
& -I_{n} \cap A_{k}=\emptyset \text { for } k<n ; \\
& -d_{u}\left(A_{n}, t_{n}\right)>0 ; \\
& -\mu\left(A_{n}\right)<\left|J_{n}\right| / 4^{n} .
\end{aligned}
$$

For a construction of such $I_{n}, J_{n}$ and $A_{n}$ it suffices to find a nowhere dense closed set

$$
A_{n} \subset \mathbb{R} \backslash \bigcup_{k \neq n}\left\{t_{k}\right\} \backslash \bigcup_{k<n} A_{k}
$$

such that $d_{u}\left(A_{n}, t_{n}\right)>0$ and next fix some closed intervals $I_{n}$ and $J_{n}$ satisfying all requirements. 
Denote by $B_{n}$ the set of all points $t \in A_{n}$ at which $d_{u}\left(A_{n}, t\right)>0$. Let

$$
f_{\alpha}(x)= \begin{cases}f\left(t_{n}\right) & \text { if } x \in B_{n}, n=1,2, \ldots \\ 0 & \text { otherwise on } \mathbb{R}\end{cases}
$$

Then $f_{\alpha} \in \mathcal{A}_{2}$ and $f=\lim _{\alpha} f_{\alpha}$.

Theorem 11. If functions $f_{\alpha} \in \mathcal{A}_{4}$ for $\alpha<\omega_{1}$ and $f=\lim _{\alpha} f_{\alpha}$, then $f \in \mathcal{A}_{4}$.

Proof. Assume, by a contrary, that $f$ is not in $\mathcal{A}_{4}$. Since by Theorems 1,9 and Remark 9 the function $f \in \mathcal{A}_{1}$, there are a positive real $\eta$, a point $x$ and a set $A \in \mathcal{T}_{d}$ such that $x \in A$ and for every open interval $I$ with $I \cap A \neq \emptyset$ and $f(I \cap A) \subset(f(x)-\eta, f(x)+\eta)$ there is a point $t \in I \cap A$ at which the function $f$ is not approximately continuous. Let $\left(x_{n}\right)_{n}$ be a sequence of points such that the set $\left\{\left(x_{n}, f\left(x_{n}\right) ; n=1,2, \ldots\right\}\right.$ is dense in the graph of the function $f$ and for each open interval $I$ with $I \cap A \neq \emptyset$ and $f(I \cap A) \subset(f(x)-\eta, f(x)+\eta)$ there is a point $x_{n(I)} \in I \cap A$ at which $f$ is not approximately continuous. There is a countable ordinal number $\beta$ such that $f_{\alpha}\left(x_{n}\right)=f\left(x_{n}\right)$ for all countable ordinal numbers $\alpha \geq \beta$ and $n=1,2, \ldots$. Consequently, the functions $f$ and $f_{\beta}$ are almost everywhere equal. Since $f \in \mathcal{A}_{1}$, there is an open interval $I$ such that $I \cap A \neq \emptyset$ and $f(A \cap I) \subset(f(x)-\eta, f(x)+\eta)$. From the relation $f_{\beta} \in \mathcal{A}_{4}$ we obtain that there is an open interval $J \subset I$ such that $J \cap A \neq \emptyset$, $f_{\beta}(J \cap A) \subset(f(x)-\eta, f(x)+\eta)$ and the function $f_{\beta}$ is approximately continuous at every point $t \in J \cap A$. Let a point $u=x_{k} \in A \cap J$ be such that the function $f$ is not approximately continuous at $u$. Since $f_{\beta}(u)=f(u)$ and the functions $f$ and $f_{\beta}$ are equal at almost all points, the function $f_{\beta}$ must be approximately continuous at $u$, a contradiction.

Observe that there are Baire 1 functions which are not in $\mathcal{A}_{6}$. Since every Baire 1 function is the limit of a transfinite sequence of approximately continuous functions (see [7]), then the classes $\mathcal{A}_{i}, i=6,7$, are not closed under the transfinite convergence. But if a function $f$ is the limit of a transfinite sequence of functions $f_{\alpha} \in \mathcal{A}_{6}\left(f_{\alpha} \in \mathcal{A}_{7}\right)$, then $f$ is pointwise discontinuous (pointwise discontinuous on each nonempty set belonging to $\mathcal{T}_{d}$ ).

Problem. Characterize the functions being the limits of transfinite sequences of functions belonging to $\mathcal{A}_{i}, i=6,7$.

\section{References}

[1] A. M. Bruckner, Differentiation of real functions, Lectures Notes in Math.659 (1978), Springer-Verlag. 
[2] R. O. Davies, Approximate continuity implies measurability, Proc. Cambridge Philos. Soc. 73 (1973), 461-465.

[3] Z. Grande, La mesurabilité des fonctions de deux variables et de la superposition $F(x, f(x))$, Dissert. Math. 159 (1978), 1-50.

[4] Z. Grande, Measurability, quasicontinuity and cliquishness of functions of two variables, Real Analysis Exch. 20 No.2 (1994-95), 744-752.

[5] Z. Grande, On strong quasi-continuity of functions of two variables, Real Analysis Exch. 21 No.1 (1995-96), 236-243.

[6] Z. Grande, Sur la quasi-continuité et la quasi-continuité approximative, Fund. Math. 129 (1988), 167-172.

[7] J. S. Lipiński, On transfinite sequences of mappings, Cas. pestovani matem. 101 (1976), 153-158.

[8] R. D. Mauldin, The Baire order of the functions continuous almost everywhere, Proc. Amer. Math. Soc. 41 (1973), 535-540.

[9] T. Neubrunn, Quasi-continuity, Real Anal. Exch. 14, No. 2, (1988-89), 259-306. 
